\title{
Psoriasis-associated genetic polymorphism in North Indian population in the CCHCR1 gene and in a genomic segment flanking the HLA-C region
}

\author{
G. Gandhi ${ }^{\mathrm{a}}$, B. Singh Buttar ${ }^{\mathrm{a}}$, L. Albert ${ }^{\mathrm{b}}$, Q. Hasan ${ }^{\mathrm{c}}$ and R.K. Aggarwal ${ }^{\mathrm{b}, *}$ \\ ${ }^{a}$ Department of Human Genetics, Guru Nanak Dev University, Amritsar, India \\ ${ }^{\mathrm{b}}$ Centre for Cellular and Molecular Biology (CSIR), Hyderabad, India \\ ${ }^{\mathrm{c}}$ Department of Genetics and Molecular Medicine, Kamineni Hospitals, Hyderabad, India
}

\begin{abstract}
Psoriasis is a common, chronic, recurrent, inflammatory, hyper proliferative disorder of the skin, which has a relatively high prevalence in the general population (0.6-4.8\%). Linkage and association analyses in various populations have revealed a major locus for psoriasis susceptibility, PSORS1, at 6p21.3. Association of the disease with human leukocyte antigen (HLA) Cw6, corneodesmosin (CDSN) and the coiled-coil alpha-helical rod protein-1 (CCHCR1) has also been reported. Though the PSORS1 locus accounts for 30-50\% of familial psoriasis in various global population groups, yet no studies have been published from the North Indian population. Some of the SNPs in HLA-C and CCHCR1 genes have been reported as markers for disease susceptibility. Therefore in the present study, DNA samples from psoriasis patients from North India were genotyped for polymorphisms in CCHCR1 and HLA-C genes. The allele frequencies were calculated for patients and controls, and were compared for odds ratio and confidence interval values. SNPn.7*22222 (rs12208888), SNPn.7*22333 (rs12216025), SNPn.9*24118 (rs10456057), CCHCR1_386 (rs130065), CCHCR1_404 (rs130076) and CCHCR1_1364 (rs130071) were found to be significant in psoriasis patients. Linkage disequilibrium analysis revealed two haplotypes $(r s 12208888, r s 2844608, r s 12216025, r s 10456057, r s 130065$, rs 130066, rs 130068, rs130269, and rs12208888, rs2844608, rs12216025, rs130076, rs130066, rs 130068, rs130269, rs130071) as highly susceptible haplotypes for psoriasis in the cohort studied. Preliminary analysis of the data also suggests the possibilities of ethnic group specific disease related polymorphisms, pending validation in future studies.
\end{abstract}

Keywords: Psoriasis, SNP Polymorphism, North-Indian, CCHCR1 gene, HLA-C gene

\section{Introduction}

Psoriasis (MIM *177900) is one of the most prevalent immune-mediated skin diseases in adults occurring at a frequency of $1-2 \%$ among Caucasians and with a lower frequency $(0.1 \%)$ in Asians [6,7]. In India, the prevalence of psoriasis has recently been inferred as varying from 0.44 to $2.80 \%$ [14]. The disease is due to

\footnotetext{
*Corresponding author: Ramesh K Aggarwal, Ph.D., FNAAS, FAPSc, Chief Scientist, Group Leader, W 409-Molecular Genetics Lab. Centre for Cellular and Molecular Biology (CSIR-CCMB), Uppal Road, Hyderabad 500 007, India. Tel.: +91 4027192635 (direct); 2716022240 (EPBX); E-mail: rameshka@ccmb.res.in.
}

the keratinocyte hyper proliferation, and is characterized by red scaly lesions either localized or widespread in extent on the extensor surfaces. The patients have the typical silvery white scales, which vary in numbers. Psoriasis may be erythrodermic, palmo-plantar, vulgaris, inverse type or guttate, albeit the most common phenotype is psoriasis vulgaris accounting for $80-90 \%$ of the cases [23].

Genetic-environmental interactions have been proposed as a cause of psoriasis. Twin and family studies suggest a heritability of $60-90 \%$. It is a genetically heterogeneous condition $[8,15,17,20]$. Genomewide scans have indicated that 10-20 chromosomal regions harbour psoriasis susceptibility genes; further- 
more, genetic predisposition is also suggested in studies indicating linkage to the Human Leukocyte Antigens (HLA) [16,19,20,24,26-29].

Several psoriasis susceptibility loci (PSORS1-9) have been mapped to different chromosomes. Among these, PSORS1 at $6 \mathrm{p} 21.3$ has been proposed to be the major candidate locus along with HLA complex [20, 37]. A study in 171 nuclear families revealed a 10 $\mathrm{kb}$ core risk haplotype as the PSORSI susceptibility locus near to HLA-C [37]. This PSORS1 gene has been reported in different ethnic groups. An association of the disease has also been reported with human leukocyte antigen Cw6 and other genes like, corneodesmosin (CDSN) and the coiled-coil alpha-helical rod protein-1 (CCHCR1). Overall the PSORS1 disease susceptible region contains three genes: HLAC (Human Leukocyte Antigen-C; OMIM *142840), CCHCR1 and CDSN. HLA-Cw*6 allele is involved with psoriasis-related immune response and has been observed as a significant marker for psoriasis risk prediction [1,11]. Based on both, population- and familybased association analyses of haplotypes and by exclusion analysis of recombinant haplotypes in Hans Chinese, Fan et al. [18] refined the PSORS1 gene to a region with HLA-C as was suggested originally by Nair et al. [30].

The CCHCR1 (OMIM*605310) gene located 110 $\mathrm{kb}$ telomeric to HLA-C consists of 18 exons stretching over $14.7 \mathrm{~kb}$. The CCHCR1 gene is highly polymorphic and one of its alleles, CCHCR $1 *$ WWCC, is suggested to be directly related to the keratinocyte proliferation [35]. Asumalahti et al. [3] identified two SNPs in CCHCR1 that were associated significantly with psoriasis in Finnish families. They also observed an over expression of CCHCR1 gene in keratinocytes of psoriatic lesions implying a potential role of this in the pathogenesis of psoriasis. Genotyping of 419 psoriasis families further revealed that CCHCR $1 *$ WWCC (four SNPs, 2 each in exons 4 and 10) and HLA-Cw*6 allele were associated with psoriasis [4].

A genome scan of the $220-\mathrm{kb}$ region (at chromosome $6 \mathrm{p} 21$ ) across 171 family-based trios of European origin, identified 59 SNPs (18 in coding and 41 in non-coding regions), of which two SNPs (n.7 and n.9 lying 7 and $4 \mathrm{~kb}$ proximal to HLA-C, respectively) were found to exhibit highly significant association $\left(p<10^{-9}\right)$, suggesting these two SNPs as markers for psoriasis susceptibility [37]. This observation was also replicated in a Gujarati Indian case/control study [4]. More recently, Chang et al. [12] explored the above two SNPs of the CCHCR 1 gene, along with HLA-CW*0602 by direct sequencing for disease association and showed that the CCHCR1 gene's SNP n.7*A and SNP n.9*C, as well as, $\mathrm{CW}^{*} 0602$ were major susceptibility markers for psoriasis in Chinese patients.

Most of these studies showing PSORS1 association with the disease have been based on European/Caucasian and Chinese populations. There is no study from India or information available for Indian populations, except for one European study wherein some subjects of Indian Gujarati descent were also analyzed [10]. Therefore, the present study was carried out to ascertain the association(s), if any, by genotyping markers representing HLA-C region (SNPs n.7, n.9) and CCHCR1 gene (exons 4 and 10) with psoriasis in Indian patients mainly from North India (visiting a local medical institute in Amritsar, Punjab), and a few cases from Hyderabad, Andhra Pradesh.

\section{Materials and methods}

A case-control study design was used and the control group was adequately matched with the patient group. A record of the population sub-structure was also maintained. To reduce the effect of population stratification (confounding by ethnicity), family-based controls were also used [25]; though, these are also not without bias, because of over- matching and environmentsharing [34].

Psoriasis patients $(n=44)$ and controls ( $n=28 ; 18$ were normal healthy controls and 10 were positive controls viz. first-degree relatives of psoriasis patients from Punjab) were investigated for genetic polymorphismdisease association(s).

\subsection{Subjects}

Psoriasis patients were contacted from Skin and Tuberculosis ward, Sri Guru Ram Das Institute of Medical Sciences and Research Charitable Hospital, Amritsar during July- Dec.2005. In addition, three patients were from Kamineni Hospitals Ltd., Hyderabad, representing North Indians living in Andhra Pradesh. All the patients were diagnosed by the dermatologists but were not allotted any Psoriasis severity index (PASI) score. In each case, blood samples were collected after informed written consent. The study was approved by the Institutional Ethics Committee of each of the collaborating institutes. 
Table 1

Primers used in the present study, designed from NCBI sequences: AC004195 (CCHCR1 Gene) and AC004204 (HLA-C region)

\begin{tabular}{lllc}
\hline Primer ID & \multicolumn{1}{c}{ Target region } & 5'-3' Sequence & Amplicon size (bp) \\
\hline Pso1F & HLA-C SNP n. 7 & GCACCACATTTTCTTTATCCATCTATA & 272 \\
Pso1R & & TGGTGAGGCTGTGGAGAAATAGGAAC & \\
Pso2F & HLA-C SNP n. 9 & GTCCACGGGGCTTTCATGATTATAC & 376 \\
Pso2R & & TCCAAACTGAAATGCTCTTACTCCTC & \\
Pso3F & CCHCR1 Exon-4 & GGTCCTCTTTTCCTTCTCCTGGCTATT & 445 \\
Pso3R & & GCCCAGCTCTCCGTTATGAATTTGAA & \\
Pso4F & CCHCR1 Exon-10 & GCCGCAGAGGTGGAGGTGGAG & 420 \\
Pso4R & & CCCCAGGAAAAGAAACGAACACAGGC & \\
\hline
\end{tabular}

\subsection{Methods}

Intravenous blood samples were obtained by venipuncture for all subjects in EDTA $(0.5 \mathrm{M})$ vaccutainers, and transported to the laboratory on ice for molecular analysis. Whole blood samples were processed to obtain genomic DNA using standard procedures. DNA samples were quantified and checked by agarose gel electrophoresis.

For each of the test domains, the primer pairs were designed from the database retrieved sequences of CCHCR1 gene (GenBank Accession no. AC004195) and HLA-C region (GenBank Accession no. AC0042 04) using Gene Tool software (www.biotools.com), and custom synthesized by Bioserve, Hyderabad, India (Table 1). These primers were named as: Pso1F/R, Pso2F/R for the HLA-C and Pso3F/R, Pso4F/R for CCHCR1 gene to target amplicon sizes of $272 \mathrm{bp}, 376$ bp, 445 bp, 420 bp, respectively (Table 1). All primers were designed such (long lengths and high GC content) that the PCR could be carried out under relatively higher stringency/annealing temperature. Further, each of the amplicon was sequenced for both the strands. These measures were taken to avoid the possible noise from the non-target HLA regions having nearly similar sequences.

\subsection{PCR amplification}

PCR amplifications were carried out in $15 \mu \mathrm{l}$ reactions, each containing $\sim 5.0 \mathrm{ng}$ of genomic DNA, 5 pmol of forward/reverse primer, $150 \mu \mathrm{M}$ dNTPs, $1 \mathrm{x}$ PCR buffer [500 mM KCl, $100 \mathrm{mM}$ Tris- $\mathrm{HCl} \mathrm{pH}$ 8.3], $1.5 \mathrm{mM} \mathrm{MgCl}_{2}$ and 1 unit Taq DNA polymerase in PTC-200 thermal cycler (MJ Research). The amplification profile comprised an initial denaturation step of $95^{\circ} \mathrm{C}$ for $3 \mathrm{~min}$, followed by 36 three-step cycles of: denaturation at $94^{\circ} \mathrm{C}$ for $45 \mathrm{sec}$, annealing at $57^{\circ} \mathrm{C}$ for 1 min, extension at $72^{\circ} \mathrm{C}$ for $2 \mathrm{~min}$, and a final extension at $72^{\circ} \mathrm{C}$ for $5 \mathrm{~min}$. The PCR products were checked on $1.5 \%$ agarose gel before sequencing.

\subsection{Sequencing}

PCR products obtained were directly sequenced for both the strands by fluorescent di-deoxy-terminator chemistry (BigDye, Applied Biosystems) following manufacturer's instructions on an ABI PRISM 3700 automated DNA sequencer. A total of 288 nucleotide sequences (obtained for the 72 study subjects for the four domains) were deposited in the NCBI core nucleotide database under accession numbers EF055578 to EF055865. These sequences were then looked for polymorphisms by comparing with the genomic reference using the Auto assembler software (ABI, Applied Biosystems).

\subsection{Statistical analyses}

Statistical analyses were carried out with the help of GOLD Software (http://www.sph.umich.edu/csg/abeca sis/gold/index.html; for linkage disequilibrium -D' values) and Medcalc Software (www.MedCalc.be) for odds ratio, CI (Confidence Interval) values and p-values. Bonferroni correction was also used to get stringent $\mathrm{p}$ values.

\section{Results}

There were 27 psoriasis male patients with mean age of $38.90 \mathrm{y}(7-80 \mathrm{y})$ and 17 female patients with mean age of 38.50 y $(25-60 y)$, whereas controls $(n=28)$ comprised 16 males with mean age of $35.60 \mathrm{y}$ and 12 females with mean age of $34.54 \mathrm{y}$ (Table 2). Among the patients, there were 41 with psoriasis vulgaris and three palmo-plantar cases. Early onset $(\leqslant 40 \mathrm{y})$ of the disease was predominant $(n=35)$ than the late $(>40 \mathrm{y})$ onset for which there were nine patients (Table 2).

\section{Study of polymorphic sites}

Seventy-two subjects were analyzed by sequencing for the four polymorphic sites of the HLA-C region 
Table 2

Clinical Characteristics of Psoriasis Patients

\begin{tabular}{|c|c|c|c|c|c|c|c|}
\hline \multicolumn{2}{|c|}{ Characteristics/range } & \multicolumn{3}{|c|}{ Patients } & \multicolumn{3}{|c|}{ Controls } \\
\hline & & Males & Females & Total & Males & Females & Total \\
\hline \multirow{2}{*}{ Age (years) } & $\leqslant 30$ & $12(27.27)^{*}$ & $6(13.64)$ & $18(40.90)$ & $11(39.29)$ & $5(17.86)$ & $16(57.14)$ \\
\hline & $31 \leqslant 80$ & $15(34.09)$ & $11(25.00)$ & $26(59.09)$ & $5(17.86)$ & $7(25.00)$ & $12(42.86)$ \\
\hline \multirow{2}{*}{ Age- of- onset (years) } & $<40$ & $21(47.55)$ & $14(13.82)$ & $35(79.55)$ & - & - & \\
\hline & $\geqslant 40$ & $6(13.64)$ & $3(6.82)$ & $9(20.41)$ & - & - & \\
\hline Distortion of nails & Present & $9(20.45)$ & $5(11.36)$ & $14(31.82)$ & - & - & \\
\hline Seasonal severity & Summer & $26(59.09)$ & $16(36.36)$ & $42(95.45)$ & - & - & \\
\hline Family history & Present & $6(25.00)$ & $5(11.36)$ & $11(36.36)$ & - & - & \\
\hline \multirow[t]{2}{*}{ Type of Psoriasis } & Psoriasis vulgaris & $26(59.09)$ & $15(34.09)$ & $41(93.18)$ & - & - & \\
\hline & Palmoplantar Psoriasis & $1(2.22)$ & $2(4.55)$ & $3(6.82)$ & - & - & \\
\hline \multirow{4}{*}{ Population Sub-groups } & Jat Sikh & $11(25.00)$ & $10(22.73)$ & $21(47.73)$ & $5(17.86)$ & $6(21.43)$ & $11(39.29)$ \\
\hline & Ramgharhia & $3(6.82)$ & $1(2.27)$ & $4(9.09)$ & $4(14.29)$ & $2(7.14)$ & $6(21.43)$ \\
\hline & Brahmin & $10(22.73)$ & $3(6.82)$ & $13(29.55)$ & $4(14.29)$ & $4(14.29)$ & $8(28.57)$ \\
\hline & Majbhi Sikh & $3(6.82)$ & $3(6.82)$ & $6(13.64)$ & $3(10.71)$ & - & $3(10.71)$ \\
\hline
\end{tabular}

* Number of subjects; value in parenthesis is (\%).

(SNPs n.7 and n.9) and CCHCR1 gene (exons 4, 10), thus resulting in a total of 288 nucleotide sequences [NCBI accession numbers EF055578 to EF055865]. Target and/or novel SNPs, and genotypes significantly varying from control are presented in Table 3.

\subsection{HLA-C region}

The region was found to be highly polymorphic; a total of 30 polymorphic sites were observed out of which 22 sites were present around SNPn.7 and eight sites around SNPn.9. Out of these, four sites $\left(22211^{*} \mathrm{G}\right.$, $22222 * \mathrm{~A}, 22238 * \mathrm{G}, 22333 * \mathrm{G}$ ) around SNPn. 7 and two sites $(24118 * \mathrm{C}, 24134 * \mathrm{~T})$ around SNPn.9 were associated with disease phenotype. The sites $22222 * \mathrm{~A}$ (rs12208888) and $22333^{*} \mathrm{G}$ (rs12216025) in SNPn.7 were significantly associated (Tables 4,5 ). The allele $22222 *$ A (rs12208888) was $42.04 \%$ in patients vs. $21.42 \%$ in controls and was significantly associated with psoriasis (OR 2.66; CI 1.23-5.72, $p \leqslant$ $0.01)$. Interestingly, a similar significant $(p \leqslant 0.01 \mathrm{lev}-$ el) association was seen in patients for the $22333 * \mathrm{G}$ (rs12216025) allele. Likewise, of the two sites in SNPn.9, only $24118^{*} \mathrm{C}$ (rs10456057) had a significant odds ratio of 7.06 (CI 2.56-19.42; $p \leqslant 0.001$ ). The allele frequency in patients was $40.91 \%$ and $8.93 \%$ in controls (Table 4).

\subsection{CCHCRl gene}

This region was less polymorphic compared to the HLA-C region and exhibited a total of 11 polymorphic sites: 6 in exon 4 and 5 in exon 10.

\subsubsection{Exon 4}

Five out of six sites (CCHCR1_384*A, CCHCR1_ 386*T, CCHCR1_404*T, CCHCR1_556*G, CCHCR1_
$571 * \mathrm{C})$ were associated with psoriasis but only $\mathrm{CCHC}$ R1_386*T and CCHCR1_404*T (rs130065, rs130076) showed a statistically significant association (Table 5). Genotypic analysis at CCHCR1_386*T (rs130065) revealed an allele frequency of $37.50 \%$ in patients and $10.71 \%$ in controls (OR 5.00; CI value 1.93-12.93, at $p \leqslant 0.001)$. The $\mathrm{C}$ to $\mathrm{T}$ alteration changes the amino acid from normal Arg to Trp. The allele frequency at the CCHCR1_404*T (rs130076) in patients was also higher $(44.30 \%)$ than in controls $(10.71 \%)$ and highly significant with odds ratio 6.63 (CI value 2.57-17.07, $p \leqslant 0.001)$.

\subsubsection{Exon 10}

Four of the five sites (CCHCR1_1328*C, CCHCR1_ 1329*A, CCHCR1_1354*T and CCHCR1_1364*C) in this exon showed association with psoriasis; of this $1364 * \mathrm{C}$ (rs130071) was higher $(48.86 \%)$ and significant (OR 3.16, CI 1.49-6.67) in patients when compared to controls $(23.21 \%)$.

\subsection{Linkage disequilibrium}

Linkage disequilibrium is the non-random association of alleles at linked loci and can be calculated as the coefficient of linkage disequilibrium, D; this is the difference between the observed and expected haplotype frequency under statistical independence depending on allele frequencies. In the present study, a total of 15 markers forming part of HLA-C region (4 in SNP n.7 and 2 in SNP n.9) and of CCHCR1 gene (5 in exon 4 and 4 in exon 10) were studied. In order to calculate inter-marker linkage disequilibrium, the Graphical Overview of Linkage Disequilibrium (GOLD) software was used. Distances between 


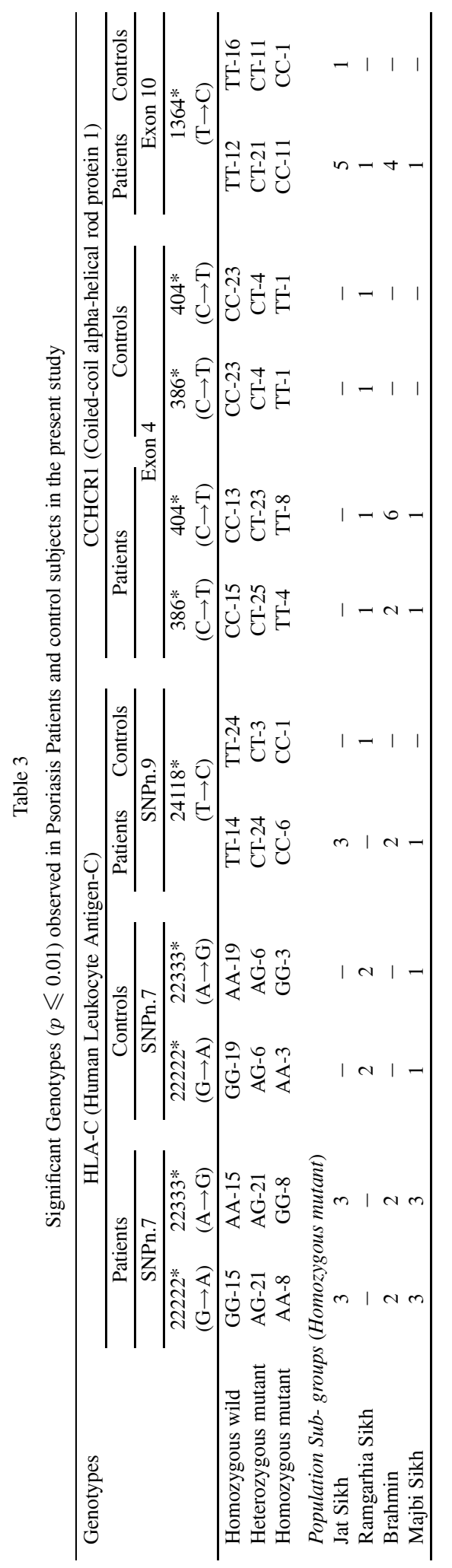


Table 4

Allele Frequencies of the HLA-C Region SNPs (n.7 and n.9) in Psoriasis Patients and Control Subjects

\begin{tabular}{|c|c|c|c|c|c|c|}
\hline Allele & \multicolumn{2}{|c|}{ Patients } & Controls & odds ratio & $95 \%$ CI value & p-value \\
\hline \multicolumn{7}{|l|}{ SNPn.7 } \\
\hline \multirow[t]{2}{*}{$22211 * \mathrm{~A} \rightarrow \mathrm{G}(\mathrm{rs} 2844607)$} & ALL & $89.70 \%(79 / 88)$ & $85.71 \%(48 / 56)$ & 1.46 & $0.53-4.04$ & 0.463 \\
\hline & Early Onset & $91.43 \%(64 / 70)$ & & 1.77 & $0.58-5.46$ & 0.315 \\
\hline \multirow[t]{2}{*}{$22222 * \mathrm{G} \rightarrow \mathrm{A}(\mathrm{rs} 12208888)$} & ALL & $42.00 \%(37 / 88)$ & $21.42 \%(12 / 56)$ & 2.66 & $1.23-5.72$ & 0.012 \\
\hline & Early Onset & $48.57 \%(34 / 70)$ & & 3.46 & $1.56-7.64$ & $0.002 *$ \\
\hline \multirow[t]{2}{*}{$22238^{*} \mathrm{~A} \rightarrow \mathrm{G}(\mathrm{rs} 2844608)$} & ALL & $69.30 \%(61 / 88)$ & $66.07 \%(37 / 56)$ & 1.16 & $0.56-2.37$ & 0.683 \\
\hline & Early Onset & $70.00 \%(49 / 70)$ & & 1.19 & $0.56-2.54$ & 0.638 \\
\hline \multirow[t]{2}{*}{$22333 * \mathrm{~A} \rightarrow \mathrm{G}(\mathrm{rs} 12216025)$} & ALL & $42.00 \%(37 / 88)$ & $21.43 \%(12 / 56)$ & 2.66 & $1.23-5.72$ & 0.012 \\
\hline & Early Onset & $48.57 \%(34 / 70)$ & & 3.46 & $1.56-7.64$ & $0.002 *$ \\
\hline \multicolumn{7}{|l|}{ SNPn.9 } \\
\hline \multirow[t]{2}{*}{$24118 * \mathrm{~T} \rightarrow \mathrm{C}(\mathrm{rs} 10456057)$} & ALL & $40.91 \%(36 / 88)$ & $8.93 \%(5 / 56)$ & 7.06 & $2.56-19.42$ & $0.0002 *$ \\
\hline & Early Onset & $41.40 \%(29 / 70)$ & & 8.10 & $2.88-22.76$ & $0.0001^{*}$ \\
\hline \multirow[t]{2}{*}{$24134 * \mathrm{G} \rightarrow \mathrm{T}$ (novel) } & ALL & $12.50 \%(11 / 88)$ & $3.57 \%(2 / 56)$ & 3.85 & $0.82-18.10$ & 0.087 \\
\hline & Early Onset & $14.28 \%(10 / 70)$ & & 4.50 & $0.94-21.45$ & 0.059 \\
\hline
\end{tabular}

Significant $\mathrm{p}$-values $(\leqslant 0.05)$ are highlighted in bold; ${ }^{*}$ Statistical significance was also established after Bonferroni correction.

Table 5

Allele distribution of CCHCR1 SNPs in Psoriasis Patients and Control Subjects

\begin{tabular}{|c|c|c|c|c|c|c|}
\hline Allele & \multicolumn{2}{|c|}{ Patients } & Controls & odds ratio & $95 \%$ CI value & p-value \\
\hline \multicolumn{7}{|l|}{ Exon 4} \\
\hline \multirow[t]{2}{*}{ CCHCR1_384*A (rs130075) } & ALL & $3.40 \%(3 / 88)$ & $3.57 \%(2 / 56)$ & 0.95 & $0.15-5.88$ & 0.958 \\
\hline & Early Onset & $2.85 \%(2 / 70)$ & - & 0.79 & $0.10-5.82$ & 0.820 \\
\hline \multirow[t]{2}{*}{ CCHCR1_386*A (rs130065) } & ALL & $37.50 \%(33 / 88)$ & $10.71 \%(6 / 56)$ & 5.00 & $1.93-12.93$ & $0.0009^{*}$ \\
\hline & Early Onset & $41.42 \%(29 / 70)$ & - & 5.89 & $2.23-15.56$ & $\mathbf{0 . 0 0 0 3}^{*}$ \\
\hline \multirow[t]{2}{*}{ CCHCR1_404*A (rs130076) } & ALL & $44.30 \%(39 / 88)$ & $10.71 \%(6 / 56)$ & 6.63 & $2.57-17.07$ & $0.0001^{*}$ \\
\hline & Early Onset & $47.14 \%(33 / 70)$ & - & 7.43 & $2.82-19.57$ & $0.0001^{*}$ \\
\hline \multirow[t]{2}{*}{ CCHCR1_556*A (rs130077) } & ALL & $5.68 \%(5 / 88)$ & $0 \%(0 / 56)$ & - & - & - \\
\hline & Early Onset & $4.28 \%(3 / 70)$ & - & - & - & - \\
\hline \multirow[t]{2}{*}{ CCHCR1_571*A (rs130066) } & ALL & $20.45 \%(18 / 88)$ & $16.07 \%(9 / 56)$ & 0.54 & $0.25-1.16$ & 0.116 \\
\hline & Early Onset & $22.85 \%(16 / 70)$ & - & 0.62 & $0.28-1.37$ & 0.245 \\
\hline \multicolumn{7}{|l|}{ Exon 10} \\
\hline \multirow[t]{2}{*}{ CCHCR1_1328*C (rs130068) } & ALL & $37.50 \%(33 / 88)$ & $51.78 \%(29 / 56)$ & 0.55 & $0.28-1.10$ & 0.092 \\
\hline & Early Onset & $35.71 \%(25 / 70)$ & - & 0.51 & $0.25-1.05$ & 0.071 \\
\hline \multirow{2}{*}{ CCHCR1_1329*A (rs130269) } & ALL & $12.50 \%(11 / 88)$ & $8.92 \%(5 / 56)$ & 1.45 & $0.477-4.44$ & 0.508 \\
\hline & Early Onset & $15.71 \%(11 / 70)$ & - & 1.90 & $0.61-5.83$ & 0.261 \\
\hline \multirow[t]{2}{*}{ CCHCR1_1354*T (rs130070) } & ALL & $2.27 \%(2 / 88)$ & $5.35 \%(3 / 56)$ & 0.41 & $0.06-2.54$ & 0.338 \\
\hline & Early Onset & $0 \%(0 / 70)$ & - & - & - & - \\
\hline \multirow[t]{2}{*}{ CCHCR1_1364*C (rs130071) } & ALL & $48.86 \%(43 / 88)$ & $23.21 \%(13 / 56)$ & 3.16 & $1.49-6.67$ & $0.002 *$ \\
\hline & Early Onset & $51.42 \%(36 / 70)$ & - & 3.50 & $1.60-7.62$ & 0.001* \\
\hline
\end{tabular}

Significant p-values $(\leqslant 0.05)$ are highlighted in bold; ${ }^{*}$ Statistical significance was also established after Bonferroni correction.

the markers were calculated in base pairs with the help of reference sequence from the NCBI database (accession number BA000025.2), which covers all the SNPs together. On the basis of significant D' values, nine haplotypes were generated in control individuals and 14 haplotypes in patients which were highly significant (Figs 1,2). Though individual allele frequencies may not increase the disease risk but in combination they may contribute to the development of Psoriasis. In the controls, only very few alleles were in linkage disequilibrium with each other. This may be due to the fact that a few controls were first-degree relatives having similar parental origin. Hence the markers showing LD may be the susceptible alleles in this patient group or generally, individuals with these alleles have a higher chance to be affected with psoriasis.

\section{Ethnicity and psoriasis}

The subjects (patients and controls) analyzed in the present study comprised four different ethnic groups, of which the largest group was of the Jat Sikhs (Table 2). Hence, an attempt was made to see if any of the observed polymorphisms were associated with ethnicity. Interestingly, all the three observed HLA-C SNPs n.7 and n.9 polymorphisms i.e., 22222G*A, 22333A*G, $24118 \mathrm{~T} * \mathrm{C}$ (rs12208888, rs12216025, rs10456057) 


\begin{tabular}{|c|c|c|c|c|c|c|c|c|c|c|c|c|c|c|c|c|}
\hline \multicolumn{2}{|r|}{ SNPs } & 1 & 2 & 3 & 4 & 5 & 6 & 7 & 8 & 9 & 10 & 11 & 12 & 13 & 14 & 15 \\
\hline 1 & $\begin{array}{l}22211^{*} A \rightarrow G \\
(\mathrm{rs} 2844607)\end{array}$ & & 1.000 & 1.000 & 1.000 & 0.137 & 1.000 & 0.167 & 0.060 & 0.336 & 0.301 & 0.022 & 0.036 & 1.000 & 0.611 & 0.034 \\
\hline 2 & $\begin{array}{l}22222^{*} G \rightarrow A \\
(r s 12208888)\end{array}$ & & & 1.000 & 1.000 & 0.900 & 0.539 & 1.000 & 0.628 & 0.404 & 1.000 & 0.075 & 0.178 & 0.460 & 1.000 & 0.128 \\
\hline 3 & $\begin{array}{l}22238^{*} A \rightarrow G \\
(\mathbf{r s 2 8 4 4 6 0 8 )}\end{array}$ & & & & 1.000 & 0.861 & 0.653 & 1.000 & 0.651 & 0.607 & 1.000 & 0.118 & 0.036 & 0.298 & 0.410 & 0.149 \\
\hline 4 & $\begin{array}{l}22333^{*} A \rightarrow G \\
(\text { rs12216025) }\end{array}$ & & & & & 0.900 & 0.539 & 1.000 & 0.628 & 0.404 & 1.000 & 0.075 & 0.178 & 0.460 & 1.000 & 0.128 \\
\hline 5 & $\begin{array}{l}24118 * T \rightarrow C \\
(\text { rs10456057) }\end{array}$ & & & & & & 1.000 & 1.000 & 0.569 & 0.350 & 1.000 & 0.185 & 0.092 & 0.378 & 0.996 & 0.207 \\
\hline 6 & $\begin{array}{l}24134^{*} G \rightarrow T \\
\text { (novel) }\end{array}$ & & & & & & & 0.567 & 1.000 & 0.601 & 0.494 & 0.086 & 0.087 & 0.103 & 1.000 & 0.284 \\
\hline 7 & $\begin{array}{l}\text { CCHCRI } 384 * A \\
(\text { (rs130075) }\end{array}$ & & & & & & & & 1.000 & 1.000 & 1.000 & 1.000 & 0.640 & 1.000 & 1.000 & 1.000 \\
\hline 8 & $\begin{array}{l}\text { CCHCRI } 386^{*} A \\
\text { (rs130065) }\end{array}$ & & & & & & & & & 1.000 & 1.000 & 0.556 & 0.733 & 1.000 & 0.640 & 0.408 \\
\hline 9 & $\begin{array}{l}\text { CCHCR1 } 404^{*} A \\
\text { (rs130076) }\end{array}$ & & & & & & & & & & 1.000 & 0.499 & 0.626 & 1.000 & 0.999 & 0.353 \\
\hline 10 & $\begin{array}{l}\text { CCHCR1 } 556^{*} A \\
\text { (rs130077) }\end{array}$ & & & & & & & & & & & 0.022 & 0.574 & 0.086 & 0.633 & 0.313 \\
\hline 11 & $\begin{array}{l}\text { CCHCR1 } 571 * A \\
\text { (rs130066) }\end{array}$ & & & & & & & & & & & & 0.289 & 0.429 & 0.162 & 0.432 \\
\hline 12 & $\begin{array}{l}\text { CCHCRI } 1328^{*} \mathrm{C} \\
\text { (rs130068) }\end{array}$ & & & & & & & & & & & & & 1.000 & 1.000 & 0.844 \\
\hline 13 & $\begin{array}{l}\text { CCHCR1_1329*A } \\
\text { (rs130269) }\end{array}$ & & & & & & & & & & & & & & 1.000 & 1.000 \\
\hline 14 & $\begin{array}{l}\text { CCHCRI } 1354^{*} T \\
\text { (rs130070) }\end{array}$ & & & & & & & & & & & & & & & 1.000 \\
\hline 15 & $\begin{array}{l}\text { CCHCR1 } 1364 * C \\
(\text { rs130071) }\end{array}$ & & & & & & & & & & & & & & & \\
\hline
\end{tabular}

Fig. 1. Intermarker-Linkage Disequilibrium (GOLD software) between Single Nucleotide Polymorphism (SNP) from HLA-C Region and CCHCR1 Gene in Psoriasis Patients.

were significantly associated with psoriasis in patients of the ethnic groups: Jat Sikhs, Brahmins and Majbi Sikhs, but none in the Ramgarhia Sikhs. A similar ethnic propensity was apparent for the disease-related polymorphisms observed only in the exon- 4 and not in the exon-10 of CCHCR1 gene. Disease- related polymorphisms CCHCR1_386C*T and CCHCR1_404C*T (rs130065, rs130076) were seen only in Brahmin and Majbi Sikh patients; none in Jat Sikhs and, equally in patient/control samples of Ramgarhia Sikhs (Table 3).

\section{Discussion}

Psoriasis, an immune-mediated skin disease, is suggested to be highly heritable and genetically heterogeneous [8,15-17,20]. A perusal of a number of fine mapping studies on different global populations suggests a critical region of about $300 \mathrm{~kb}$ for PSORS1, containing HLA-C and a few other genes/critical regions viz., a 285-kb region between the markers tn 821 and HLA-C [5]; a 111-kb interval telomeric to HLA-C in the Japanese patients [31]; a 150-kb region telomeric to HLA-C in Jewish population [27]; a 46-kb interval telomeric to HLA-C in Caucasian and Japanese populations [24]; a 70-kb region around the CDSN gene in a Sardinian population [32]; a haplotype block harboring HLA-C but distinct from CDSN and CCHCR1 [21], and a 224-kb region in an American Caucasian population [30]. HLA-Cw*0602 was recognized as significantly associated with Psoriasis and as the marker that confers the highest risk for the disease [3,26].

In the present study, psoriasis patients were investigated for four susceptible markers in the PSORS1 region i.e., HLA-C region (SNPs n.7 and n.9) and CCHCR1 gene (exons 4 and 10). Direct sequence based polymorphism analysis revealed that the sites SNPn.7*_22222, SNPn.7*_22333, SNPn.9*_24118 of HLA-C region and $-386,-404$ and -1364 of CCHCR1 gene were significantly associated in the psoriasis patients tested in this study (Table 3).

The results from the present study are generally in agreement with the published studies. The PSORS1 locus contributes a 35-50\% relative risk of developing familial psoriasis $[9,29,36]$ and HLA association analyses also support the importance of the PSORS1 locus in psoriasis susceptibility, especially its tight association with the HLA-Cw*6 allele.

Genome analysis of 220-kb region at chromosome 6p21 identified two HLA-C SNPs n.7 and n.9 as highly significant $\left(p<10^{-9}\right)$ markers for disease susceptibility in Finnish population, and some individuals of Gujarati Indian descent [37]. Polymorphisms in CCHCR1 gene, as well as HLA-C region (SNP n. $7^{*} \mathrm{~A}$, SNP n.9*C, CW*0602) as major susceptibility markers 


\begin{tabular}{|c|c|c|c|c|c|c|c|c|c|c|c|c|c|c|c|c|}
\hline \multicolumn{2}{|r|}{ SNPs } & 1 & 2 & 3 & 4 & 5 & 6 & 7 & 8 & 9 & 10 & 11 & 12 & 13 & 14 & 15 \\
\hline 1 & $\begin{array}{l}22211^{*} A \rightarrow G \\
(\boldsymbol{r s} 2844607)\end{array}$ & & 1.000 & 1.000 & 1.000 & 0.826 & 1.000 & 0.311 & 1.000 & 1.000 & 0.000 & 0.611 & 1.000 & 0.829 & 1.000 & 0.038 \\
\hline 2 & $\begin{array}{l}22222^{*} G \rightarrow A \\
(r s 12208888)\end{array}$ & & & 1.000 & 1.000 & 0.210 & 1.000 & 1.000 & 0.800 & 0.800 & 0.000 & 0.222 & 0.576 & 0.408 & 1.000 & 0.419 \\
\hline 3 & $\begin{array}{l}22238^{*} A \rightarrow G \\
(\mathbf{r} \mathbf{2} 2844608)\end{array}$ & & & & 1.000 & 0.154 & 0.993 & 1.000 & 0.509 & 0.509 & 0.000 & 0.018 & 0.375 & 0.154 & 1.000 & 0.120 \\
\hline 4 & 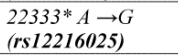 & & & & & 0.210 & 1.000 & 1.000 & 0.800 & 0.800 & 0.000 & 0.222 & 0.576 & 0.408 & 1.000 & 0.419 \\
\hline 5 & $\begin{array}{l}24118^{*} T \rightarrow C \\
(r s 10456057)\end{array}$ & & & & & & 1.000 & 1.000 & 0.260 & 0.260 & 0.000 & 0.116 & 0.302 & 0.029 & 1.000 & 0.381 \\
\hline 6 & $\begin{array}{l}24134^{*} G \rightarrow T \\
\text { (novel) }\end{array}$ & & & & & & & 1.000 & 1.000 & 1.000 & 0.000 & 0.263 & 1.000 & 1.000 & 0.450 & 0.068 \\
\hline 7 & $\begin{array}{l}\text { CCHCR1 } 384 * A \\
\text { (rs130075) }\end{array}$ & & & & & & & & 1.000 & 1.000 & 0.000 & 0.263 & 0.999 & 1.000 & 1.000 & 0.068 \\
\hline 8 & $\begin{array}{l}\text { CCHCR1 } 386^{*} A \\
\text { (rs130065) }\end{array}$ & & & & & & & & & 1.000 & 1.000 & 1.000 & 1.000 & 0.162 & 1.000 & 0.429 \\
\hline 9 & 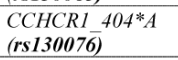 & & & & & & & & & & 0.000 & 1.000 & 1.000 & 0.162 & 1.000 & 0.429 \\
\hline 10 & $\begin{array}{l}\text { CCHCR1 556*A } \\
\text { (rs130077) }\end{array}$ & & & & & & & & & & & 0.000 & 0.000 & 0.000 & 0.000 & 0.000 \\
\hline 11 & $\begin{array}{l}\text { CCHCR1 } 571 * A \\
\text { (rs130066) }\end{array}$ & & & & & & & & & & & & 0.034 & 0.378 & 0.018 & 0.043 \\
\hline 12 & $\begin{array}{l}\text { CCHCRI } 1328 * C \\
\text { (rs130068) }\end{array}$ & & & & & & & & & & & & & 1.000 & 1.000 & 0.661 \\
\hline 13 & $\begin{array}{l}\text { CCHCRI_1329*A } \\
\text { (rsl30269) }\end{array}$ & & & & & & & & & & & & & & 1.000 & 0.487 \\
\hline 14 & $\begin{array}{l}\text { CCHCRI } 1354 * T \\
\text { (rs130070) }\end{array}$ & & & & & & & & & & & & & & & 1.000 \\
\hline 15 & $\begin{array}{l}\text { CCHCRI_1364*C } \\
\text { (rs130071) }\end{array}$ & & & & & & & & & & & & & & & \\
\hline
\end{tabular}

Fig. 2. Intermarker-Linkage Disequilibrium (GOLD software) between Single Nucleotide Polymorphism (SNP) from HLA-C Region and CCHCR1 Gene in Control Individuals.

for psoriasis were also indicated in a Chinese population [12].

Haplotype-based association analysis revealed SNP n. $7 *$ A-SNPn9*C-Cw*0602-CCHCR1_386*T-CCHC R1_404*T-CCHCR1_1802*T-CCHCR12406*G as a major susceptibility haplotype. Two of the SNPs $(\mathrm{CCH}$ CR1_386*T, CCHCR1_404*T) observed in the Chinese study [12] were previously indicated as susceptible SNPs in a Finnish population [3]. In subsequent studies Asumalahti et al. [2,4] demonstrated an association of $\mathrm{CCHCR} 1 * \mathrm{WWCC}$ with psoriasis and also with the HLA-Cw*6 allele. They reported association of HLA-CW*6, CCHCR $1 *$ WWCC, and CDSN*5 genes with clinical variants of psoriasis (guttate psoriasis and palmoplantar psoriasis); but they did not observe any apparent correlation with the age of onset for disease. Moreover, their analysis suggested that psoriasis vulgaris and guttate psoriasis have a similar genetic basis (albeit the latter exhibits a stronger association with PSORSI susceptibility alleles: HLA-CW*6, CCHCR1*WWCC, and CDSN*5) while palmoplantar psoriasis appeared to be a distinct disorder.

There were three palmo-plantar cases and 35 patients with early age-of-onset in the present study (Table 2). The data for these subjects when compared with the remaining patients/controls, suggest an apparent, significant association of psoriasis in early onset patients with HLA-C region (rs12208888, rs12216025 in SNP n.7 and rs10456057 in SNP n.9) and CCHCR1 gene (rs130065, rs130076 in exon 4 and rs130068, rs130071 in exon 10). Similarly, in the palmoplantar cases, the data suggest an association with homozygous risk alleles: $22211 * \mathrm{G}, 22238 * \mathrm{~A}$ (rs12208888, rs2844608) and $404 * \mathrm{C}, 571 * \mathrm{C}, 1328 * \mathrm{~T}, 1364 * \mathrm{~T}$ (rs130076, rs13 0066, rs130068, rs130071), and heterozygous alleles: $22222^{*} \mathrm{G}, 22333^{*} \mathrm{~A}, 24118 * \mathrm{~T}(\mathrm{rs} 12208888$, rs 122 16025 , rs 10456057$)$ and $386 * \mathrm{C}, 1328 * \mathrm{~T}, 1329 * \mathrm{G}$, $1364 * \mathrm{~T}$ (rs130065, rs130068, rs130269, rs130071), of HLA-C and CCHCR1 genes, respectively. (The early onset ( $<40$ years) patients had severe psoriasis while milder symptoms were present in late onset patients. Strong HLA associations in type I psoriasis and much weaker HLA association in late onset ( $\geqslant 40$ years) has been documented [12,13,22,33].

In the present study, Linkage disequilibrium test (D' values) revealed two haplotypes viz. SNPn.7_22222* A-SNPn.7_22238*G-SNPn.7_22333*G-SNPn.9_241 18*G-CCHCR1_386*T-CCHCR1_571*C_CCHCR1_ $328 * \mathrm{C}-\mathrm{CCHCR} 1 \_329 * \mathrm{~A}$ (rs 12208888, rs2844608, rs 1 2216025, rs10456057, rs130065, rs 130066, rs 130068, rs 130269) and SNPn.7_22222*A-SNPn.722238*GSNPn.7_22333*G-CCHCR1_404*T-CCHCR1_571*C -CCHCR1_328*C-CCHCR1_329*A-CCHCR1_364* $\mathrm{T}$ (rs12208888, rs2844608, rs 12216025, rs130076, rs 130066, rs130068, rs130269, rs130071), as highly susceptible major haplotypes for psoriasis in the cohort 
studied. The alleles found significant in the present study were also associated in the Chinese population [12], with the exception of SNPn.7_22211*G (rs28 44607) and SNPn.7_22238*G (rs2844608) which did not show any association with psoriasis in the Indian patients, and the allele CCHCR1_1364*T (rs130071) which was significant in the present study but not in the Chinese patients.

The people of North India are predominantly IndoAryan, and include various ethnic groups such as Jats, Rajputs, Gujjars, Ahirs, Khatris, Kambojs, Banias and Dalits. Sikhs who are mainly found in the Punjab region of India refer to themselves as Jat Sikhs, Mazabhi Sikhs, Ramgarhia Sikhs (http://www.hindubooks. org/sudheer_birodkar/hindu_history/castevedic.html). A critical look at the data obtained in the present study suggests apparent associations between some of the identified disease-related polymorphisms of both HLA$\mathrm{C}$ and CCHCR1 genes with some of the ethnic group(s) analyzed in this study. Although, the significance of these observations is limited due to the small sample size, yet these provide interesting possibilities of developing ethnic- group- specific diagnostic/genetic markers of psoriasis. This would need further extended validation studies with larger sample size.

Given the genetic heterogenous etiology of psoriasis and gene-environment interactions, more exhaustive research would be required to identify stable biomarkers and realize their potential in disease prediction and/or personalized medicine that is coming of age. Similarly, exhaustive functional studies are needed to determine the mechanism of action of the disease-causing candidate genes [19], including that of the CCHCR1 protein in psoriasis pathogenesis.

\section{Acknowledgements}

We are indebted to the psoriasis patients and controls who participated in this study, to Dr. P.S. Girgila (of Sri Guru Ram Das Institute of Medical Sciences and Research Charitable Hospital, Amritsar) for diagnosing the patients, Dr. Badaruddoza for helping with data analysis, and the Director, CCMB (CSIR) for permission to use DNA sequencing facilities. This work was supported by departmental grants from GNDU.

\section{Electronic-Database Information}

Online Mendelian Inheritance in Man (OMIM), http: //www.ncbi.nlm.nih.gov/Omim/ (for psoriasis [MIM*
177900], HLA-C [MIM *142840] and CCHCR1 [MIM *605310]. A set of 288 nucleotide sequences (generated for the 72 study subjects in the study) are deposited in the NCBI sequence database with accession numbers- EF055578 to EF055865.

\section{References}

[1] K.S. Amr, A.H. Weshahy, El Hadidi, F. Mahmoud, A. El Hamid et al., A Pilot Study of HLA-Cw6 Association with Egyptian Psoriatic Patients, J Medicine Medical Sci 5 (2010), 25-29.

[2] K. Asumalahti, M. Ameen, S. Suomela, E. Hagforsen, G. Michaëlsson et al., Genetic analysis of PSORS1 distinguishes guttate psoriasis and palmoplantar pustulosis, J Invest Dermatol 120 (2003), 627-632.

[3] K. Asumalahti, T. Latinen, M.L. Itkonen-Vatjus, R Lokki, S. Suomela et al., A candidate gene for psoriasis near HLA-C, HCR (Pg8), is highly polymorphic with a disease-associated susceptibility allele, Hum Mol Genet 9 (2000), 1533-1542.

[4] K. Asumalahti, C. Veal, T. Laitinen, S. Snomela, M. Allen et al., Coding haplotype analysis supports HCR as the putative susceptibility gene for Psoriasis at the MHC PSORSI locus, Hum Mol Genet 11 (2002), 589-597.

[5] N. Balendran, R.L. Clough, J.R. Arguello, R. Barber, C. Veal et al., Characterization of the major susceptibility region for psoriasis at chromosome 6p21.3, J Invest Dermatol 113 (1999), 322-328.

[6] A.M. Bowcock, The Genetics of Psoriasis and Auto-immunity, Ann Rev Genomics Human Genetics 6 (2005), 93-122.

[7] A.M. Bowcock and W.O.C.M. Cookson, The genetics of psoriasis, psoriatic arthritis and atopic dermatitis, Hum Mol Genet 13 (2004), R43-R55.

[8] F. Brandrup, N. Holm, N. Grunnet, K. Henningsen and H.E. Hansen, Psoriasis in monozygotic twins: variations in expression in individuals with identical genetic constitution, Acta Derm Venereol 62 (1982), 229-236.

[9] A.D. Burden, S. Javed, M. Bailey, M. Hodgins, M. Connor and D. Tillman, Genetics of psoriasis: paternal inheritance and a locus on chromosome 6p, J Invest Dermatol 110 (1998), 958-960.

[10] F. Capon, I.K. Toal, J.C. Evans, M.H. Allen, S. Patel et al., Haplotype analysis of distantly related populations implicates corneodesmosin in psoriasis susceptibility, J Med Genet 40 (2003), 447-452.

[11] L. Carlen, K. Sakuraba, M. Stahle and F. Sanchez, HLA-C expression pattern is spatial different between psoriasis and eczema skin lesions, J Invest Dermatol 127 (2007), 342-348.

[12] Y.T. Chang, Y.M. Shiao, P.J. Chin, Y.L. Liu, F.C. Chou et al., Genetic polymorphisms of the HCR gene and a genomic segment in close proximity to HLA-C are associated with patients with psoriasis in Taiwan, Br J Dermatol 150 (2004), 1104-1111.

[13] E. Christophers and T. Henseler, Psoriasis type I and type II as subtypes of nonpustular psoriasis, in: Psoriasis, (2nd ed.), H. Roenigk and H. Maibach, eds, Marcel Dekker Inc., New York, 1990, pp. 15-21.

[14] S. Dogra and S. Yadav, Psoriasis in India: prevalence and pattern, Ind J Dermatol Venereol Leprol 76 (2010), 595-601.

[15] D.L. Duffy, L.S. Spelman and N.G. Martin, Psoriasis in Australian Twins, J Am Acad Dermatol 29 (1993), 428-434. 
[16] J.T. Elder, A.T. Bruce, J.E. Gudjonsson, A. Johnston, P.E. Stuart et al., Molecular dissection of psoriasis: integrating genetics and biology, J Invest Dermatol 130 (2010), 12131226.

[17] J.T. Elder, R.P. Nair, T. Henseler, S. Jenisch, P. Stuart et al., The genetics of psoriasis 2001: the odyssey continues, Arch Dermatol 137 (2001), 1447-1453.

[18] X. Fan, S. Yang, W. Huang, Z.-M. Wang, L.-D. Sun et al., Fine Mapping of the Psoriasis Susceptibility Locus PSORS1 Supports HLA-C as the Susceptibility Gene in the Han Chinese Population, PLoS Genet 4(3) (2008), e1000038. doi:10.1371/journal.pgen. 1000038

[19] B.J. Feng, L.D. Sun, R. Soltani-Arabshahi, A.M. Bowcock, R.P. Nair et al., Multiple loci within the major histocompatibility complex confer risk of psoriasis, PLoS Genet 5(8) (2009), e1000606.doi:10.1371/journal.pgen.1000606.

[20] E. Giardina, C. Sinibardi and G. Novelli, The Psoriasis Genetics as a model of complex disease, Current Drug TargetsInflammation and Allergy 3 (2004), 129-136.

[21] C. Helms, N.L. Saccone, L. Cao, J.A. Daw, K. Cao et al., Localization of PSORS1 to a haplotype block harboring HLA$\mathrm{C}$ and distinct from corneodesmosin and HCR, Hum Genet 118 (2005), 466-476.

[22] T. Henseler and E. Christophers, Psoriasis of early and late onset: characterization of two types of psoriasis vulgaris, $J$ Am Acad Dermatol 13 (1985), 450-456.

[23] S.J. Holm, F. Sanchez, L.M. Carlen, L. Mallbris, M. Stahle et al., HLA-Cw*0602 associates more strongly to psoriasis in the Swedish population than variants of the novel $6 \mathrm{p} 21.3$ gene PSORS1C3, Acta Derm Venereol 85 (2005), 2-8.

[24] N. Lench, M.M. Iles, I. Mackay, R. Patel, G.S. Sagoo et al., Single-point haplotype scores telomeric to human leukocyte antigen-C give a high susceptibility major histocompatability complex haplotype for psoriasis in a caucasian population, $J$ Invest Dermatol 124 (2005), 545-552.

[25] C.A. MacRae and C.J.O.'Donnell, Genomics, in: Principles of Molecular Medicine, M.S. Runge and C. Patterson, eds, Humana Press, Totowa, New Jersey, 2006, pp. 166-174.

[26] E. Mallon, R. Newson and C.B. Bunker, HLA-Cw6 and the genetic predisposition to psoriasis: a meta-analysis of published serologic studies, J Invest Dermatol 113 (1999), 693-695.

[27] J. Martinez-Borra, C. Brautbar, S. Gonzalez, C.D. Enk, A Lopez-Vazquez et al., The region of $150 \mathrm{~kb}$ telometic to HLA-
$\mathrm{C}$ is associated with psoriasis in the Jewish population, J Invest Dermatol 125 (2005), 928-932.

$28]$ R.P. Nair, K.C. Duffin, C. Helms, J. Ding, P.E. Stuart et al., Collaborative Association Study of Psoriasis, Genomewide scan reveals association of psoriasis with IL-23 and NFkappaB pathways, Nat Genet 41(2) (2009), 199-204.

[29] R.P. Nair, T. Henseler, S. Jenisch, P. Stuart, C.K. Bichakjian et al., Evidence for two psoriasis susceptibility loci (HLA and $17 q$ ) and two novel candidate regions (16q and 20p) by genome-wide scan, Hum Mol Genet 6 (1997), 1349-1356.

[30] R.P. Nair, P.E. Stuart, I. Nistor, R. Hiremagalore, N.V. Chia et al., Sequence and haplotype analysis supports HLA-C as the psoriasis susceptibility 1 gene, Am J Hum Genet 78 (2006), 827-851.

[31] A. Oka, G. Tamiya, M. Tomizawa, M. Ota, Y. Katsuyama et al., Association analysis using refined microsatellite markers localizes a susceptibility locus for psoriasis vulgaris within a $111 \mathrm{~kb}$ segment telomeric to the HLA-C gene, Hum Mol Genet 8 (1999), 2165-2170.

[32] S. Orru, E. Giuressi, C. Carcassi, M. Casula and L. Contu, Mapping of the major psoriasis-susceptibility locus (PSORS1) in a 70-Kb interval around the corneodesmosin gene (CDSN), Am J Hum Genet 76 (2005), 164-171.

[33] G. Swanbeck, A. Inerot, T. Martinsson, J. Wahlstrom, C. Enerback et al., Age at onset and different types of psoriasis, $\mathrm{Br} \mathrm{J}$ Dermatol 133 (1995), 768-773.

[34] D.C. Thomas and J.S. Witte, Point: Population stratification: a problem for case-control studies of candidate-gene associations? Cancer Epidemiol Biomarkers Prev 11 (2002), 505512.

[35] I. Tiala, J. Wakkinen, S. Suomela, P, Puolakkainen, R. Tammi et al., The PSORS1 locus gene CCHCR1 affects keratinocyte proliferation in transgenic mice, Hum Mol Genet 17(7) (2008).

[36] R.C. Trembath, R.L. Clough, J.L. Rosbotham, A.B. Jones, R.D.R. Camp et al., Identification of a major susceptibility locus on chromosome $6 \mathrm{p}$ and evidence for further disease loci revealed by a two stage genome-wide search in psoriasis, Hum Mol Genet 6 (1997), 813-820.

[37] C.D. Veal, F. Capon, M.H. Allen, E.K. Health, J.C. Evans et al., Family Based Analysis using a Dense Single Nucleotide Polymorphism - Based Map Defines Genetic Variation at PSORSI, the major psoriasis susceptibility locus, Am J Hum Genet 71 (2002), 554-564. 


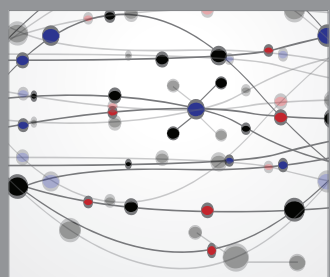

The Scientific World Journal
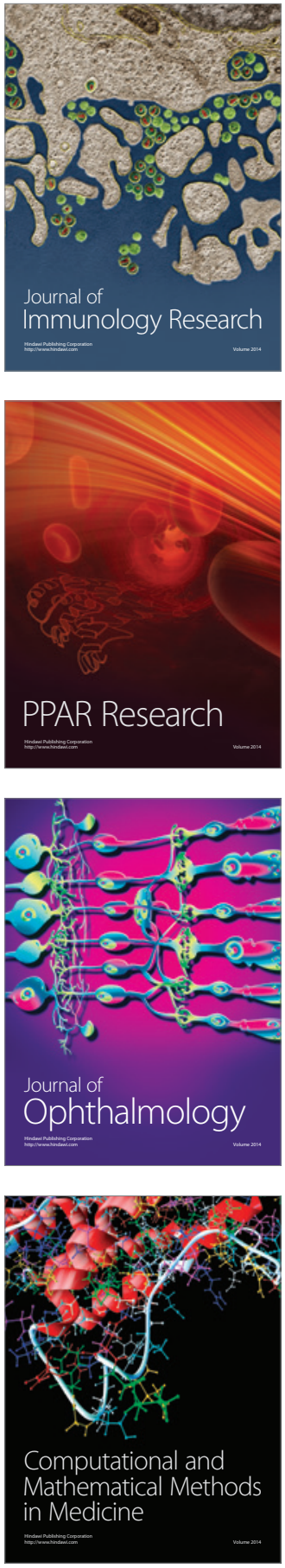

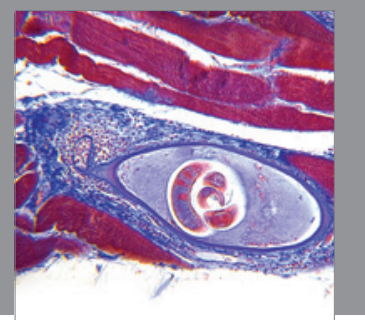

Gastroenterology

Research and Practice
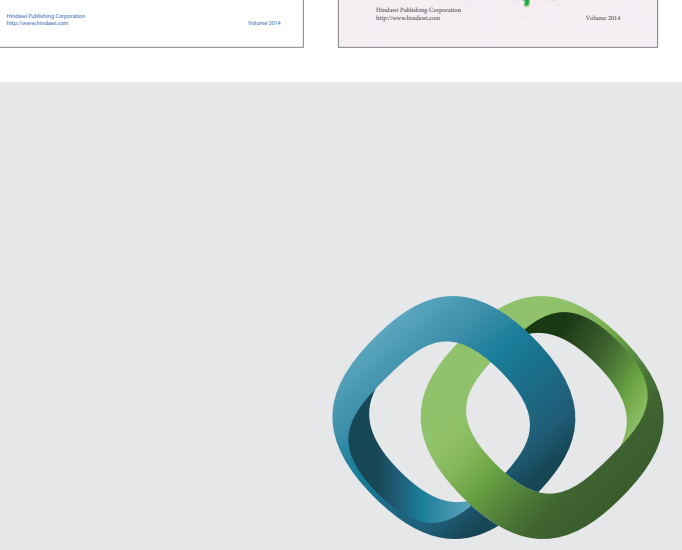

\section{Hindawi}

Submit your manuscripts at

http://www.hindawi.com
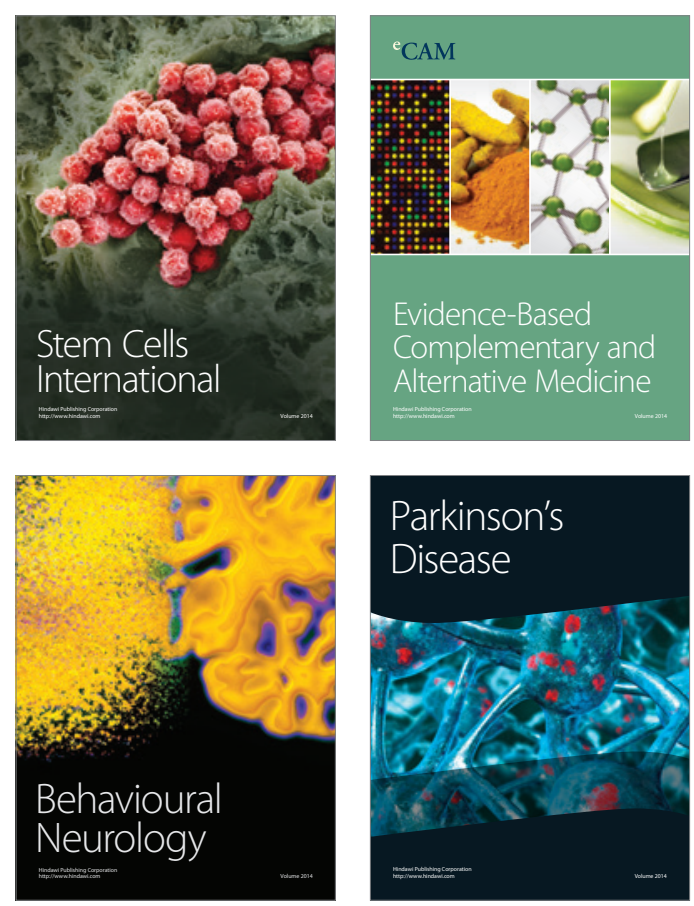

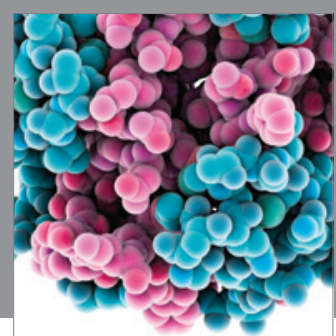

Journal of
Diabetes Research

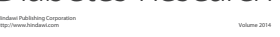

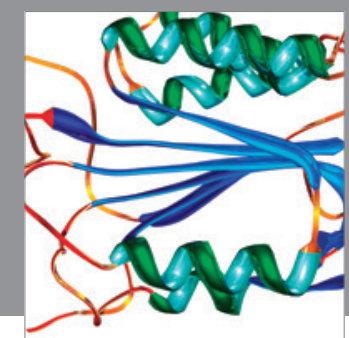

Disease Markers
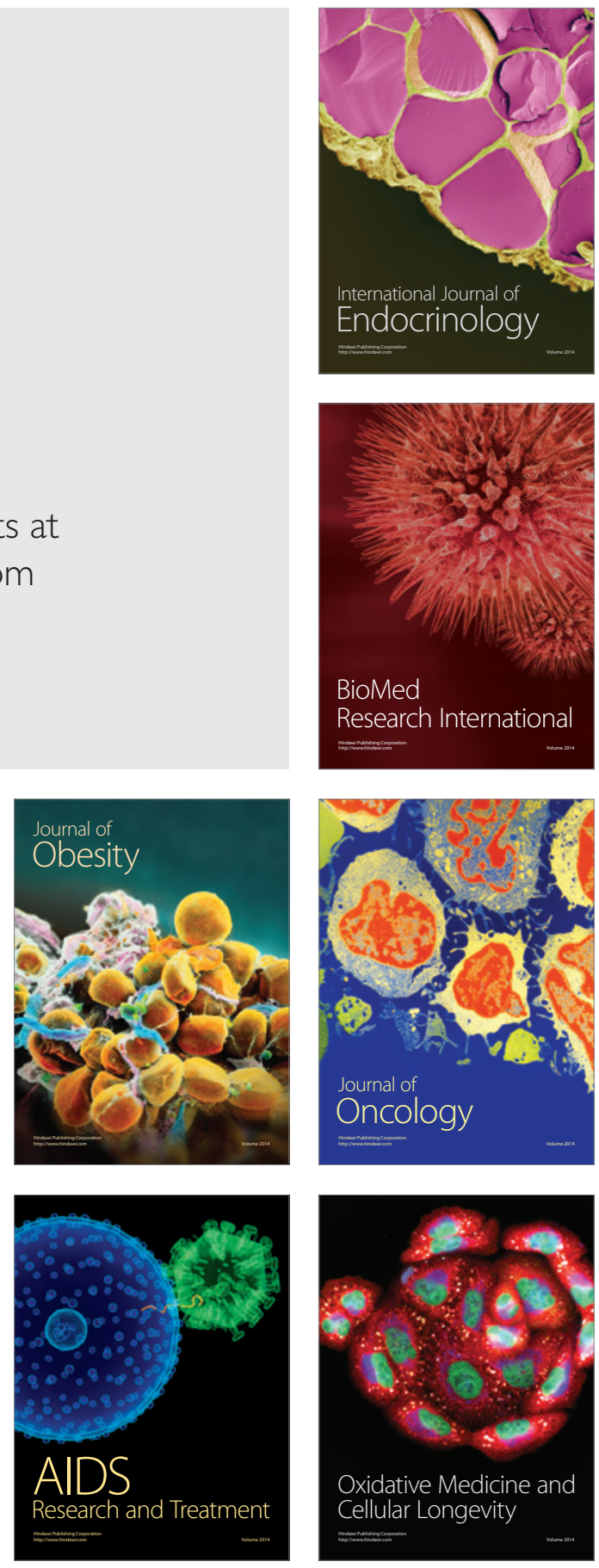